Dhaka Univ. J. Biol. Sci. 25(1): 57-64, 2016 (January)

\title{
SUICIDAL IDEATION IN RELATION TO DEPRESSION, LONELINESS AND HOPELESSNESS AMONG UNIVERSITY STUDENTS
}

\author{
Mst. Maleka Pervin and Nafiza Ferdowshi ${ }^{*}$ \\ Department of Psychology, University of Dhaka, Dhaka 1000, Bangladesh \\ Key words: Suicidal ideation, Depression, Loneliness, Hopelessness
}

\begin{abstract}
The purpose of the present study was to examine the relationship between suicidal ideation and depression, loneliness, hopelessness among University students. Study sample is comprised of 112 University students (51 males and 61 females) drawn by using purposive sampling technique. Standard questionnaires were used to obtain the data from the participants in a cross-sectional survey. The obtained data were analyzed by using Pearson product moment correlation and stepwise multiple regression. Results showed that suicidal ideation was positively correlated with depression, loneliness and hopelessness. Stepwise multiple regression analysis further reveals that depression, loneliness and hopelessness significantly predict suicidal ideation among university students. Study limitations and implications are also discussed.
\end{abstract}

\section{Introduction}

Suicide is a serious global public health problem especially among young people that can have lasting harmful effects on individuals, families, and communities. With a public health approach, prevention occurs at all levels of society from the individual, family, and community levels to the broader social environment. Effective prevention strategies are needed to promote awareness of suicide and encourage a commitment to social change. More than 800,000 people worldwide die from suicide every year. It is the 10th leading cause of death for Americans overall and the second leading cause of death among adolescents and young adults aged $15-29^{(1)}$. Suicide places a heavy burden on the nation in terms of the emotional suffering that families and communities experience as well as the economic costs associated with medical care and lost productivity. And yet suicidal behaviors often continue to be met with silence and shame. These attitudes can be formidable barriers to providing care and support to individuals in crisis and to those who have lost a loved one due to suicide.

The term "suicidal ideation" is a collective continuum from thinking about ending one's own life, to developing a plan, to a non-fatal suicide attempt, and to ending one's life. Suicide affects everyone, but some groups are at higher risk than others ${ }^{(2)}$. The range

*Author for correspondence: <nafizaf@du.ac.bd>. ${ }^{1}$ Department of Educational and Counselling Psychology, University of Dhaka, Dhaka 1000, Bangladesh. 
of suicidal ideation varies greatly from fleeting thoughts, to extensive thoughts, to detailed planning, role playing (e.g., standing on a chair with a noose), and incomplete attempt which may be deliberately constructed to not complete or to be discovered, or may be fully intended to result in death, but the individual survives e.g., for example in the case of a hanging in which the cord breaks ${ }^{(3)}$. In the United States of America, the prevalence of suicidal thoughts, suicide planning, and suicide attempts are significantly higher among young adults aged 18 - 29 years than it is among adults aged $\geq 30$ years $^{(4)}$.

Suicidal ideation means suicidal thoughts, but there are some other related risk factors which can predict suicidal ideation. Several studies have shown that suicidal ideation is associated with higher levels of depressive symptoms, hopelessness, and loneliness/isolation among young people ${ }^{(5)}$. A study conducted with Western adolescents showed risk factors associated with suicide such as hopelessness, loneliness, and cognitive, psychological, social and family factors ${ }^{(6)}$.

Suicidal ideation is generally associated with depression; however, it seems to have associations with many other psychiatric disorders, life events, and family events, all of which may increase the risk of suicidal ideation. It is recognized that major depressive disorder is a disabling condition that adversely affects a person's family life, work or school life, sleeping and eating habits, and general health conditions. In the United States of America, around $3.4 \%$ of people with major depression commit suicide, and up to $60 \%$ of people who commit suicide had depression or another mood disorder(7). In a word, depression represents the strongest and most consistent predictor of suicide among young people ${ }^{(8)}$. Konick and Gutierrez tested a path model to predict suicidal ideation in college students. Results from this study showed that depressive symptoms exerted a stronger effect than other risk factors in predicting suicidal thoughts ${ }^{(9)}$. Using a longitudinal design, Fergusson et al.(10) examined several predictors associated with suicidal ideation and suicide attempt among young people. These researchers also identified depression as the strongest predictor of suicidal behavior.

Adolescents who attempt suicide often become isolated from important people in their lives, and they experience intense feelings of isolation shortly before their attempt ${ }^{(11)}$. Moreover, many researchers have investigated loneliness as a risk factor of suicide among young people ${ }^{(12)}$. However, loneliness has been found to be a predictor of suicide ideation in early adolescence adjusting for depression, life time suicide attempts, demographics, and various measures of mental health ${ }^{(13)}$. Prinstein et al.(14) found that lower levels of close friendship support and greater levels of perceived peer rejection each directly related to suicidal ideation among adolescents. In addition, Allberg and Chu suggested that suicide risk increases for isolated adolescents, who lack support from either family or peers ${ }^{(11)}$.

Hopelessness is one of the major components of Beck's negative cognitive trait i.e. negative cognitions about future. No meeting one's own expectations and the 
expectations of significant others is a serious matter which could potentially result in loss of face which in turn leads to loss of confidence and support from one's family and these results in hopelessness. Previous research has shown that hopelessness was related to adolescent suicidal ideation. Several studies found that there was a significant relationship between hopelessness and suicidality ${ }^{(15)}$ and hopelessness was found to be the strongest of cognitive variables in concurrent association with suicidal ideation ${ }^{(16)}$. Researchers also reported that hopelessness was the best predictor of suicidal ideation in students and adolescents experiencing bipolar disorder ${ }^{(17)}$. However, there is a high association with hopelessness in long-term suicide risk.

In South Asia, Singh and Joshi ${ }^{(18)}$ examined relationship of depression, life stress and personality with suicidal ideation among college students ${ }^{(18)}$. Evidence from the previous research suggests that suicide ideation among young people is associated with a variety of factors, including depression, loneliness and hopelessness, however, research on how these factors could influence on suicidal ideation in Bangladesh is limited. A study, looks at the extent of suicide in Bangladesh, reveals the highest rate of suicide occurs between the age of 16 and 20 years and family conflict is considered as the number one reason of suicide in Bangladesh ${ }^{(19)}$. In 2011, another Bangladeshi study identifies a high positive correlation $(\mathrm{r}=0.82)$ existing between suicidal ideation and depression ${ }^{(20)}$. As this study explores the relationship of depression, loneliness and hopelessness with suicidal ideation among young people the findings of it may act as a guideline for taking further initiative and different action plan to deal with suicidal ideation. Consequently, the findings of the study may be helpful for mental health professionals to deal with young people who are suffering from depression, loneliness and hopelessness and carrying on suicidal ideation. Thus, the main purpose of the present study is to examine the association of suicidal ideation with depression, loneliness and hopelessness in Bangladesh context.

\section{Materials and Methods}

The sample for this study comprised of 112 students (51 male and 61 female) drawn from the ten different departments under 2 faculties (Biological Sciences and Arts) of the University of Dhaka by using purposive sampling strategy. The sample were taken from the Faculty of Biological Sciences that includes the Department of Psychology ( $M=15$ and $F=21)$ and Clinical Psychology $(M=5$ and $F=7)$. On the other hand, the participants were selected from the Faculty of Arts that includes the Department of English $(\mathrm{M}=4$ and $F=5)$, Bangla $(M=3$ and $F=4)$, Linguistics $(M=7$ and $F=5)$, Music $(M=4$ and $F=3)$, History $(M=3$ and $F=4)$, Philosophy $(M=4$ and $F=5)$, Arabic $(M=3$ and $F=5)$ and Islamic Studies $(\mathrm{M}=3$ and $\mathrm{F}=2)$. The age of the selected subjects ranged from 19 to 25 years (Mean 22.03 and Sd 1.52). They belong to middle socio-economic status. In general, the subjects were having good health and did not suffer from any serious or chronic ailment. 
For data collection, the present study was used the following instruments:

Demographic and personal information questionnaire: By this questionnaire, the data on age, sex, education and socio-economic status were collected as well.

Beck scale for suicide ideation: Beck scale for suicide ideation (BSS) was originally developed by Beck ${ }^{(21)}$. It consists of 19 items and each item consists of three alternative statements graded in intensity from 0 to 2 . The total scores, which range from 0 to 38, are obtained by adding the item values. The scale has reliability (Cronbach alpha) and validity coefficients of 0.89 and 0.41 , respectively ${ }^{(32)}$. Test-retest reliability co-efficient of the Bangla version of the scale was $r=0.86(p<0.01)$ and the convergent validity was $r=$ $0.78(\mathrm{p}<0.01)$ accordingly.

The depression scale: The depression scale which composed of 30 items was constructed by Rahman and Uddin for measuring depression in the context of Bangladesh (22). The highest possible score of 30 items form of depression scale was 150 and the lowest possible score was 30 . Higher score indicated higher depression and lower score indicated low level of depression.

The revised UCLA loneliness scale: The revised UCLA loneliness scale was originally developed by Russell et al.(23) for measuring loneliness including 20 items. Significant correlation $[\mathrm{r}=0.88, \mathrm{p}<0.01]$ between scores of English and Bangla version indicated translation reliability of the scale that were measured the same thing by the two versions. Thus, total scale scores can range from 20 to 80 . Higher score means high level of loneliness and lower score means low feeling of loneliness.

Beck hopelessness scale: The Beck hopelessness scale $^{(24)}$ was designed to measure negative attitudes about one's future and perceived inability to avert negative life occurrences. Beck hopelessness scale total range from 0 to 20 with higher scores indicating greater hopelessness. Test-retest reliability co-efficient of the Bangla version of the scale was $r=0.81(p<0.01)$; the split-half reliability co-efficient $r=0.59(p<0.01)$.

Standard data collection procedure was followed to collect the data from the participants. The subjects were contacted from ten different departments at the University of Dhaka for the purpose of data collection. The data collection places were also selected in accordance with the participants' convenience, such as - University campus as well as University residential areas. After getting willingness of participants, a congenial rapport was established to make them comfortable. Participants were assured of voluntary participation and that all collected data would be kept confidential. Participants were informed that they could withdraw from the study at any time. According to the 'materials' section of this article, the test tools were presented to the participants. These tests were also administered following the instructions specified in the respective test manual. The general testing conditions were satisfactory and the procedure was uniform all through. All the tests were scored as per the procedure described in respective test manual. 


\section{Results and Discussion}

Obtained data were analyzed by using correlation and stepwise regression analysis. The results are presented in Tables 1 and 2.

Table 1. Correlation co-efficient among suicidal ideation, depression, loneliness and hopelessness.

\begin{tabular}{lcccc}
\hline Measures & 1 & 2 & 3 & 4 \\
\hline Suicidal ideation & - & & & \\
Depression & $0.62^{* *}$ & - & & \\
Loneliness & $0.48^{* *}$ & $0.37^{* *}$ & - & - \\
Hopelessness & $0.71^{* *}$ & $0.65^{* *}$ & $0.59^{* *}$ & - \\
\hline
\end{tabular}

${ }^{* *} \mathrm{p}<0.01$.

The correlation matrix revealed that positive correlation was found between scores on the suicidal ideation, depression, loneliness and hopelessness, respectively.

Table 2. Regression analysis of depression, loneliness and hopelessness on suicidal ideation.

\begin{tabular}{lccc}
\hline Variables & $\mathrm{R}$ & $\mathrm{R}^{2}$ & $\mathrm{~F}$ \\
\hline Depression & 0.321 & 0.127 & $35.79^{* *}$ \\
Loneliness & 0.395 & 0.149 & $27.97^{* *}$ \\
Hopelessness & 0.423 & 0.198 & $19.23^{* *}$ \\
\hline
\end{tabular}

${ }^{* *} \mathrm{p}<0.01$.

The results of stepwise regression analysis for the dependent measure suicidal ideation indicated that three significant predictors of suicidal ideation which is significant at 0.01 probability level.

Pearson product moment correlation was used to examine the relationship of depression, loneliness and hopelessness with suicidal ideation (Table 1). The result reveals that suicidal ideation correlates positively with depression $(r=0.62, p<0.01)$ that is also consistent with Bangladesh context study ${ }^{(20)}$, loneliness $(\mathrm{r}=0.48, \mathrm{p}<0.01)$ and hopelessness $(\mathrm{r}=0.71, \mathrm{p}<0.01)$ accordingly. The significant correlation indicates that individuals who have had multiple episodes of depression are at greater risk for suicide than those who have had one episode as well as participants scoring high on depression tend to have lack of ability to overcome suicide ideas and may have plan to commit suicide. It is therefore likely that suicidal ideation further correlates positively with loneliness which indicates that strong associations among suicide ideation and different 
ways of being lonely and alone. Moreover, prevalence of suicide ideation increased with the degree of loneliness. For lonely young people who tend to have higher tendency for suicidal ideation or suicidal attempts. This finding is in keeping with prior research suggesting that loneliness is concurrently related to suicidal ideation in young people ${ }^{(12)}$. It is found that hopelessness was highly correlated with suicidal ideation. Consequently, hopelessness is one of the major components of Beck's negative cognitive triad i.e. negative cognitions about future. The expression of hopelessness in conjunction with a mental disorder represents a very dangerous warning sign like suicide attempts. Since hopelessness is positively related to adolescent suicidal ideation, it is important to cultivate the sense of hope in young people. Previous studies had found that young adults who are depressed are more likely to have suicidal ideation, as are young adults in a state of hopelessness ${ }^{(16)}$.

The stepwise multiple regressions were used to find out linear combination of different predictors of suicidal ideation. In the regression analysis depression, loneliness and hopelessness emerged as the significant predictors for suicidal ideation. The relationship between suicidal ideation and depression among the sample is notable. Research suggests that depression is a risk factor for suicide-related ideation. The multiple $\mathrm{R}$ for this variable equals to 0.321 , which suggests that depression accounts for approximately $13 \%$ of the variance $\left(R^{2}=0.127\right)$. The F-value being 35.79 which is highly significant $(\mathrm{p}<0.01)$. Loneliness appears to be another potent predictor which took entry at step two. Multiple R increased to 0.395 with the entry of loneliness in the equation after depression. The F-value being 27.97 is significant at $0.01 \%$ probability level. It means that depression and loneliness jointly account for approximately $15 \%$ of the variance in suicidal ideation. The last variable that took entry into the regression equation is hopelessness. With the entry of this variable the multiple $\mathrm{R}$ increased to 0.423 , indicating that these three variables accounted approximately $20 \%$ variance in suicidal ideation. The $\mathrm{F}$ ratio at this step equals to 19.23 which is significant at $0.01 \%$ probability level.

The results of stepwise regression analysis revealed that the linear combination of depression, loneliness and hopelessness account significant proportion of variance (20\%) in suicidal ideation among young people. This trend indicates there is a strong relationship of suicide ideation with depression, loneliness and hopelessness. The results of the present study suggest that targeting depression, loneliness and hopelessness may be as important in young adults to reduce suicidal ideation and prevent suicidal attempts.

Here, it is acknowledged that despite significant correlation with suicidal ideation, the important limitation of this study is noteworthy. The sample is small and all of them were selected from the University of Dhaka. So in future, it is necessary to conduct further studies with larger representative samples so that the study could include the 
both private and public University students for determining the relationships among these variables. Another limitation is that suicide attempts were not assessed in this study. For understanding the prevalence rate of suicidal attempts along with its relationship with depression, loneliness, and hopelessness, it is also necessary to get such information in further study. Furthermore, this study was based on data collected in a cross-sectional survey. We cannot, therefore, ascribe causality to any of the associated factors in the study. Longitudinal studies of suicidal behavior are necessary to understand the etiological pathways from protective factors to suicide prevention in University students. Overall, this study indicates a broader perspective of continuing studies in such specific area.

\section{References}

1. WHO 2014. Retrieved from http://www.who.int/mental_health/suicide-prevention/en/

2. Centers for Disease Control and Prevention 2014. Injury Prevention and Control: Violence Prevention. Retrieved from http://www.cdc.gov/ViolencePrevention/suicide/definitions. html (September 2014).

3. Gliatto MF and AK Rai 1999. Evaluation and treatment of patients with suicidal ideation. American Family Physician 59 (6): 1500-1506. PMID 10193592. Retrieved 200701-08.

4. Krug EG, LL Dahlberg, JA Mercy, AB Zwi and R Lozano 2002. World Report on Violence and Health. World Health Organization: Geneva, Switzerland. pp.185-188.

5. Lebret S, E Perret-Vaille, A Mulliez, L Gerbaud and I Jalenques 2006. Elderly suicide attempters: Characteristics and outcome. Intl. J. Geriatric Psychiatry 21: 1052-1059.

6. Page RM, J Yanagishita, J Suwanteerangkul, EP Zarco, CM Lee and NF Miao 2006. Hopelessness and loneliness among suicide attempters in school-based samples of Taiwanese, Philippine and Thai Adolescents. School Psychology International 27: 583598. http://dx.doi.org/10.1177/0143034306073415.

7. Soloff PH, KG Lynch, TM Kelly, KM Malone and JJ Mann 2000. Characteristics of suicide attempts of patients with major depressive episode and borderline personality disorder: A comparative study. Amer. J. Psychiatry 157(4): 601-608.

8. Foley D, D Goldston and E Costello 2006. Proximal psychiatric risk factors for suicidality in youth: The Great Smoky Mountains Study. Arch. Gen. Psychiatry 63: 1017-1024.

9. Konick L and P Gutierrez 2005. Testing a model of suicide ideation in college students. Suicide Life Threat Behav. 35: 181-192.

10. Fergusson D, L Woodward and L Horwood 2000. Risk factors and life processes associated with the onset of suicidal behavior during adolescence and early adulthood. Psychol. Med. 30: 23-39.

11. Allberg W and L Chu 1990. Understanding adolescent suicide: Correlates in a developmental perspective. Sch. Couns. 37: 343-350.

12. King CA and CR Merchant 2008. Social and interpersonal factors relating to adolescent suicidality: A review of the literature. Archives of Suicide Research 21: 81-196. 
13. Roberts RE, CR Roberts and YR Chen 1998. Suicidal thinking among adolescents with a history of attempted suicide. Journal of the American Academy of Child and Adolescent Psychiatry 37: 1294-1300.

14. Prinstein M, J Boergers and A Spirito 2000. Peer functioning, family dysfunction, and psychological symptoms in a risk factor model for adolescent inpatients' suicidal ideation severity. J. Clin. Child Psychol. 29: 392-405.

15. Rutter PA and AE Behrendt 2004. Adolescent suicide risk: Four psychosocial factors. Adolescence 39(154): 295-302.

16. Stewart SM, BD Kennard, PWH Lee, T Maves, C Hugues and G Emslie 2005. Hopeless and suicidal ideation among adolescents in two cultures. J. Child Psychology \& Psychiatry 46: 364-372.

17. Smith JM, LB Alloy and LY Abramson 2006. Cognitive vulnerability to depression, rumination, hopelessness, and suicidal ideation: Multiple pathways to self-injurious thinking. Suicide and Life-threatening Behavior. 36: 443-454.

18. Singh $\mathrm{R}$ and HL Joshi 2008. Suicidal ideation in relation to depression, life stress and personality among college students. Journal of the Indian Academy of Applied Psychology 34: 259-265.

19. Islam $S$ and $Z$ Borak 2012. Attitude and behavior towards suicide: Role of counseling as prevention. J. Advanced Laboratory Research in Biology 3(3): 217-223. Retrieved fromhttp://www.sospublication.co.in.

20. Sultana A, S Akhter and S Islam 2011. Relationship between suicidal ideation and depression. Bangladesh Psychological Studies. 21: 91-106.

21. Beck AT, M Kovacs and A Weissman 1979. Assessment of suicidal intention: The scale for suicide ideation. J. Consulting and Clinical Psychology 47: 343-352.

22. Rahman MM and MZ Uddin 2005. Development of a Scale of Depression for Use in Bangladesh. Bangladesh Psychological Studies 15: 25-44.

23. Russell D, LA Peplau and ML Ferguson 1978. Developing a measure of loneliness. J. Personality Assessment 42: 290-294.

24. Beck AT and RA Steer 1988. Manual for Beck Hopelessness Scale. TX: Psychological Corp, San Antonio.

(Manuscript received on 1 October, 2015; revised on 13 January, 2016) 